\title{
Dependence of Alternating Magnetic Field Intensity on Proliferation Rate of Human Breast Cancer Cell
}

\author{
Hyeji Park, Hyun Sook Lee, and Do Guwn Hwang* \\ Department of Oriental Biomedical Engineering, Sangii University, Wonju, South Korea
}

(Received 13 August 2015, Received in final form 15 September 2015, Accepted 16 September 2015)

\begin{abstract}
To investigate the effects of alternating magnetic field intensity and stimulation time on the proliferation of human breast cancer cells (BT-20), we cultured the cells under a magnetic field with a saw tooth waveform of 2 kHz. The field intensities varied from 3 to $7 \mathrm{mT}$, and the stimulation time varied from 24 to 72 hours. Cell proliferation decreased dramatically to $40 \%$ during magnetic stimulation for 72 hours at $5 \mathrm{mT}$. However, the cells were not affected by a strong magnetic field of $7 \mathrm{mT}$. The $p$-values obtained using statistical package for social science software were below 0.05 for 5-7 $\mathrm{mT}$. This means that the results have statistical significance. However, it is difficult to explain our results based on the physiology of cell membranes, which have various ionic flows at ion channels.
\end{abstract}

Keywords : alternating magnetic field, frequency dependence, cell stimulation

\section{Introduction}

Magnetic field stimulation on cells has been suggested as a promising tool for medical applications as it can affect the bioelectricity of ionic flow at the cellular membrane in a relatively non-invasive manner [1]. However, detailed protocols and methods are yet to be developed. Moreover, the systematic frequency dependence of alternating magnetic field stimulation with a constant field intensity has not yet been reported, because it is not easy to control electrically the power supply to generate a constant field in the coil as the frequency increases. In addition, it is also difficult to suppress the increase in the temperature of the coil. Most studies on the effect of magnetic field stimulation on in vivo and in vitro have been performed at extremely low frequencies with electrical appliances of $50-60 \mathrm{~Hz}$ or radio frequencies of several $\mathrm{MHz}$ to $\mathrm{GHz}[2,3]$.

Studies on the biological effects of magnetic field stimulation in the intermediate frequency range of 200 $\mathrm{Hz}-10 \mathrm{kHz}$ are important, because the response time of ionic flow in ion channels of cellular membranes is a few $10^{-3} \mathrm{sec}$ in cells excited by external stimulus. Few effects of intermediate frequency magnetic fields in human have

CThe Korean Magnetics Society. All rights reserved.

*Corresponding author: Tel: +82-33-730-0413

Fax: +82-33-738-7962, e-mail: dghwang@sangji.ac.kr been reported, and further studies are strongly needed to understand the physiological mechanism at this frequency range [4].

Xiaowen et al. studied the in vivo effects of magnetic fields on intradermal tumors in mice after subcutaneous injection of human breast adenocarcinoma cells. After four days of alternating magnetic field treatments at 400 $\mathrm{kHz}$, no tumors could be discerned on the treated side, whereas a large tumor formed on the untreated side [5]. Jin Liang et al. reported that cell death exhibited a relatively linear dependence on electric field intensity in the intermediate radio frequency range of $100 \mathrm{kHz}-1000$ $\mathrm{kHz}$ and relatively low field intensities below $10 \mathrm{~V} / \mathrm{cm}$. Tumor cell growth was prominently observed at $500 \mathrm{kHz}$ and $10 \mathrm{~V} / \mathrm{cm}$ [6].

Most of the studies focused on the use of a fixed frequency and intensity because it is hard to control changes in frequency and intensity. If we want to evaluate the frequency dependence at a fixed intensity, we have to solve the problem of coil heating as the frequency increases $[7,8]$. Therefore, it is important to develop a well-designed magnetic field exposure system capable of controlling the coil heating as the frequency increases. Here, we used an alternating magnetic field stimulation system consisting of a switched-mode power supply (SMPS) and a digitally controlled circuit that modulated the duty ratio. In this work, human breast cancer cells (BT-20) were exposed by alternating magnetic field intensities of 3-7 $\mathrm{mT}$ at the 
intermediate frequency of $2 \mathrm{kHz}$, and the stimulation time was varied from 24 to 72 hours.

\section{Meterials and Methods}

We used an alternating magnetic field stimulation system consisting of a SMPS and a digital control circuit that modulated the duty ratio. The voltage applied to the coil

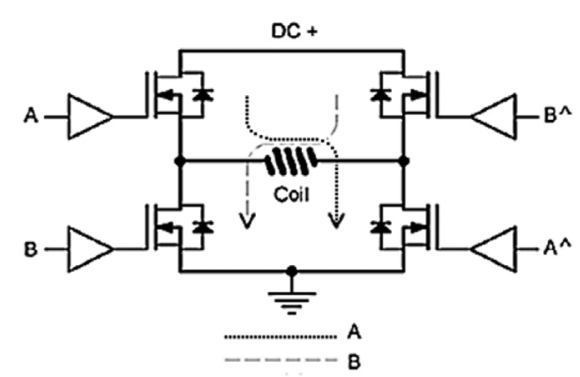

(a)

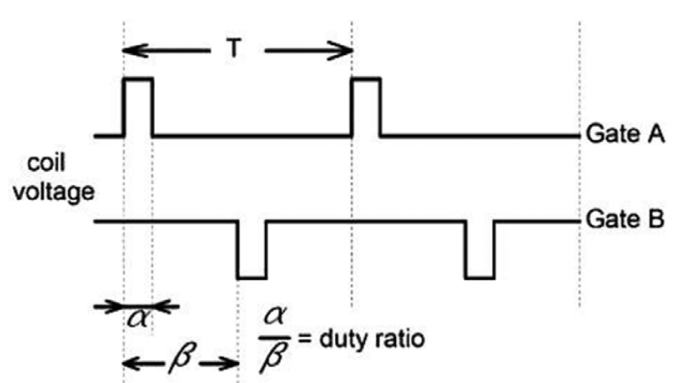

(b)

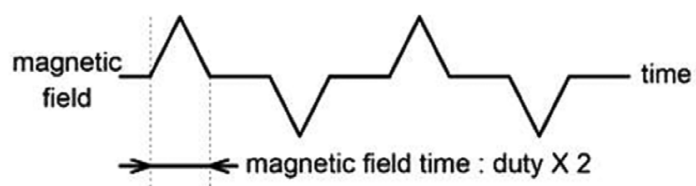

(c)

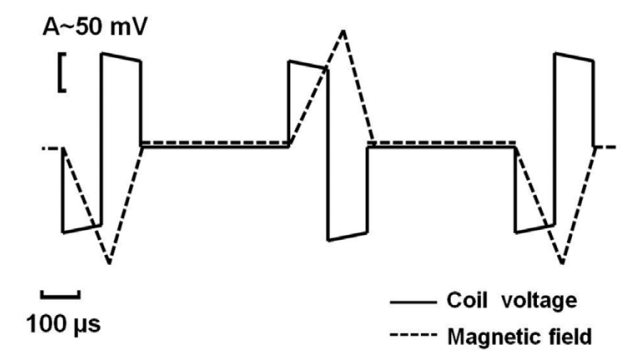

(d)

Fig. 1. (a) Schematic diagram of our designed circuit consisting of an insulated gate bipolar transistor (IGBT), (b) The coil voltage was opened by gates $\mathrm{A}$ and $\mathrm{B}$ and defined the duty factor, (c) The alternative magnetic field of the saw tooth wave type in the coil, (d) The real oscilloscope images of the applied voltage and the magnetic field measured by the second coil at $1 \mathrm{kHz}$. with increasing frequency was controlled by the duty ratio to maintain a constant magnetic field intensity of 3-7 $\mathrm{mT}$. The circuit consisted of four field transistors or insulated gate bipolar transistors, as shown in Fig. 1(a). Electric power was alternatively supplied to the coil by opening gates $\mathrm{A}$ and $\mathrm{B}$, which had electric currents flowing in opposite directions. The magnetic coil was a single layer of 15 turns with a hollow rectangular flat shape of $14 \times 6$ $\mathrm{cm}$, and the cross section of the coil wire was $1 \mathrm{~mm}$ thick and $3 \mathrm{~mm}$ wide. The 6 -well culture plate of $12.7 \times 8.5 \mathrm{~cm}$ was placed on this coil, and the water cooling system was inserted between the coil and the cell plate, as shown in Fig. 2.

Figure 1(c) shows the output voltage of the square pulse signal loaded to the coil as well as the alternative magnetic field of the saw tooth pulses measured using a digital oscilloscope and a commercial Hall sensor at 1 $\mathrm{kHz}$ in our system. To reduce the coil heating due to the increase in the impedance of $\mathbf{Z}=\left[\mathbf{R}^{2}+\boldsymbol{\omega}^{2} \mathbf{L}^{2}\right]^{1 / 2}$ as the angular frequency $\boldsymbol{\omega}$ increases, we used an alternating magnetic field based on a saw tooth wave instead of a sinusoidal wave. The power system was controlled by modulating the duty ratio to maintain a constant field intensity with increasing frequency, while the duty ratio $\boldsymbol{\alpha} / \boldsymbol{\beta}$ was defined as the ratio of pulse width $\boldsymbol{\alpha}$ and the time duration of the subsequent pulses $\boldsymbol{\beta}$ during a half cycle $\boldsymbol{T} /$

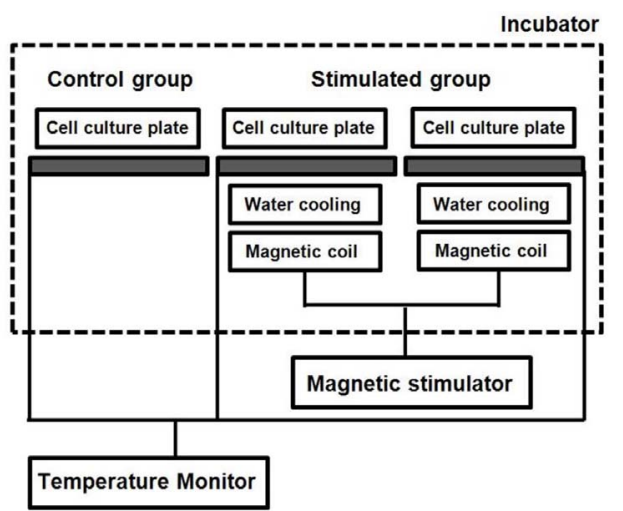

(a)

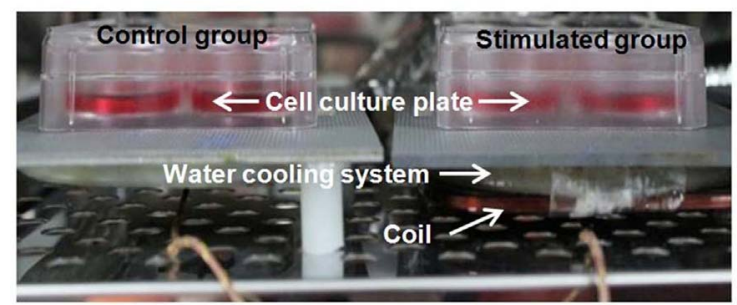

(b)

Fig. 2. (Color online) (a) Schematic diagram of alternative magnetic field stimulator, magnetic coil, water cooling system, and cell culture plates. (b) Photo image of cell culture system. 
2, as shown in Fig. 1(b). The dependence of the field intensity and frequency on the duty factor in our system was reported in previous works [9].

Our system used a SMPS to provide a maximum current of $40 \mathrm{~A}$ and a digital control circuit to generate a magnetic field of alternating saw tooth wave pulses. The magnetic field intensity was maintained at $7 \mathrm{mT}$ as the frequency was increased to $2 \mathrm{kHz}$ without coil heating. The alternating magnetic field stimulator controlled by the duty ratio and water cooling system has the advantage of preventing the incubator from exceeding the cell-viable temperature of $37^{\circ} \mathrm{C}$. The internal temperatures of the cell plate in the incubator during magnetic stimulation did not exceed $37 \pm 1.0^{\circ} \mathrm{C}$.

Human breast cancer cells (BT-20) were obtained from American Type Culture Collection and cultured in Dulbecco's Modified Eagle Medium (DMEM)/F12 (Gibco BRL, Germany) with 5\% FBS (fetal bovine serum; Gibco BRL, Germany) in a humidified atmosphere with $5 \% \mathrm{CO}_{2}$ at $37^{\circ} \mathrm{C}$. Monolayer cells were washed three times in phosphate buffered saline (PBS) and stimulated with $1 \mathrm{ng} / \mathrm{ml}$ interleukin-1 $\beta$ (IL-1 $\beta$ ) (Roche Diagnostics, Germany) in DMEM/F12 containing $0.5 \%$ lactalbumin enzyme hydrolysate (Sigma, Germany). The cells were seeded in 6 well plates and allowed to adhere for an hour. After treatments, the cells were separated with trypsin, diluted and mixed with an equal volume of Trypan blue stain. The number of viable cells excluding the dye was counted using a hemocytometer and Trypan blue assay.

After the cells attached to the culture plate, they were cultured for 24 to 72 hours under alternative magnetic field stimulation. The BT-20 cells were exposed to different intensities of $3,5,6$, and $7 \mathrm{mT}$ for 24 hours to investigate the intensity dependence of magnetic stimulation. The cells were cultured in three plates at the same time, as shown in Fig. 2(a). One plate was cultured under no stimulation as the control plate. The other two plates were cultured with magnetic stimulation under the same condition to compare the cell proliferation. If the difference in the number of cells in the two cell plates exposed to the magnetic field was above $13 \%$, we eliminated the results. The morphology and cell count were assessed with a 100X-magnification microscope (Motic, AE31).

The cell number obtained from the 6 well plate was statistically analyzed using SPSS (statistical package for social science) software. In order to confirm the statistical significance of our data, we compared the $p$-values of the cell number between the non-stimulated control cells and the cells exposed to magnetic stimulation at 3-7 $\mathrm{mT}$ using the Kruskal-Wallis test and the Mann-Whitney $U$ test [10]. In statistics, statistically significant results are attain- ed when a $p$-value is less than the significance level. The $p$-value is the probability of obtaining the more extreme results given that the null hypothesis is true whereas the significance level is the probability of rejecting the null hypothesis given that it is true. As a matter of good scientific practice, a significance level is chosen before data collection and is usually set to $0.05(5 \%)$ [11].

\section{Results and Discussion}

Human breast cancer cells were cultured over the coil generating an alternating magnetic field at $2 \mathrm{kHz}$ to study the dependence of magnetic field intensity and stimulation time on the proliferation rate of cancer cells. Before investigating the intensity effect of cell proliferation, the time required for the cell number to increase from $1 \times 10^{5}$ cells to $2 \times 10^{5}$ cells in the incubator was measured. From three experiments, we determined that BT-20 cells increased to $1.85 \times 10^{5}$ cells on average after 72 hours. If the number of control cells cultured without magnetic stimulation was below $1.6 \times 10^{5}$ cells, the result was not included in the statistical data.

Figure 3 shows the morphologies of BT- 20 cells exposed to the maximum intensities of 3,5 , and $7 \mathrm{mT}$ at the frequency of $2 \mathrm{kHz}$ for 72 hours. The non-stimulated control cells, as shown in the morphology in Fig. 3(a), grew very well and attached to the plate surface. The cells exposed to $3 \mathrm{mT}$ also grew well, but the cell growth was interrupted by stimulation at $5 \mathrm{mT}$ and the cells did not attach to the plates, as shown in Fig. 3(b) and (c). At 7 $\mathrm{mT}$, the growth and attachment of cells recovered above the amount at $5 \mathrm{mT}$. From the cell morphologies with
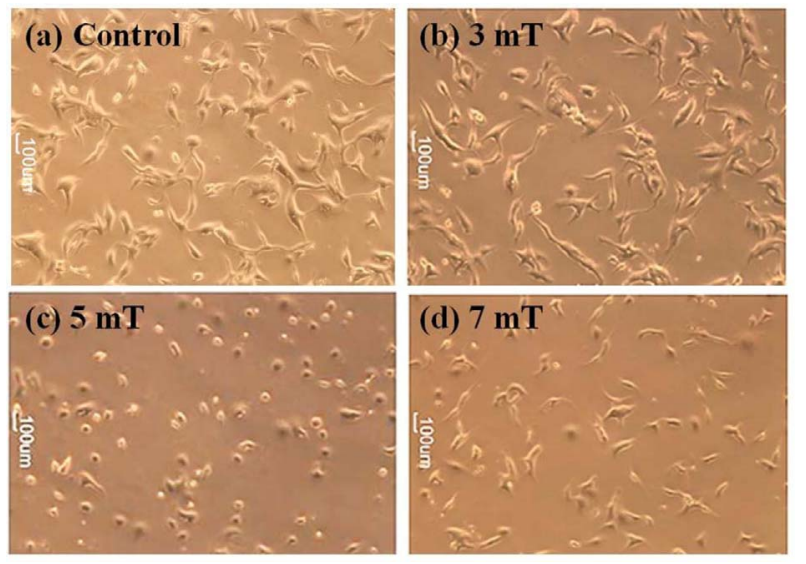

Fig. 3. (Color online) The cell morphologies of human breast cancer cells (BT-20), shows (a) the unstimulated control cells, (b) the cells exposed to magnetic field at $3 \mathrm{mT}$, (c) $5 \mathrm{mT}$, and (d) $7 \mathrm{mT}$. The cells stimulated at $5 \mathrm{mT}$ did not attach to the plate surface. 


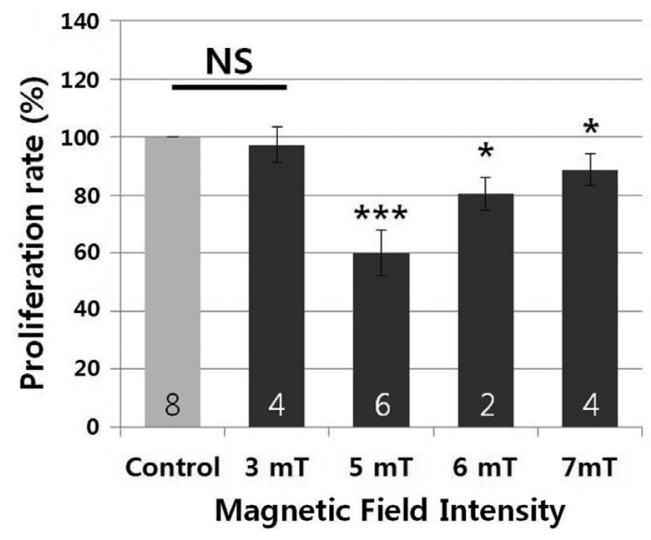

Fig. 4. Proliferation rates of BT-20 cells exposed to the magnetic field intensities of 3, 5, 6 and $7 \mathrm{mT}$ at the frequency of 2 $\mathrm{kHz}$ for 72 hours. The stimulated cells were cultured in two plates (right and left). The proliferation rate of the stimulated cells was suppressed up to $60 \%$ at $5 \mathrm{mT}$, with $p$-values of $* p<$ $0.05, * * p<0.01$, and $* * * p<0.001$ and the number noted below each bar is the number of repetitions.

various magnetic field intensities, we could find that there is an optimum condition for suppressing cancer cell proliferation. In order to elucidate the effect of the specific intensity, $5 \mathrm{mT}$, of magnetic field on BT-20 cell, further experiment for ion channel in cell membrane (i.e. two-photon $\mathrm{Ca}^{+2}$ imaging) explaining the physiological mechanism is needed.

Figure 4 shows the proliferation rates of BT-20 cell depending on magnetic field intensity. Compared to the number of non-stimulated control cells, the proliferation rate after 72 hours of stimulation at $2 \mathrm{kHz}$ at the intensities of 3,5, 6, and $7 \mathrm{mT}$ were calculated. At $3 \mathrm{mT}$, the proliferation ratios varied from $90 \%$ to $103 \%$, with an average ratio of $97 \%$. However the proliferation of breast cancer cells was suppressed to $60 \%$ at $5 \mathrm{mT}$, with a standard deviation of 7.74. With an increase in the magnetic field intensity to $7 \mathrm{mT}$, the cell proliferation was increased to $88 \%$.

Table 1 shows the statistical analysis of the cell number for the non-stimulated control cells and the stimulated cells at alternating magnetic fields of 3, 5, 6, and $7 \mathrm{mT}$. To obtain reliable data for cell proliferation, the stimulated cells were cultured in two plates, named the right and left cells. At $3 \mathrm{mT}$, the experiment was repeated two times, and the cell numbers for the control groups ranged from $1.67-1.88 \times 10^{5}$. The cell numbers for the stimulated groups varied slightly at $1.57-1.95 \times 10^{5}$. The average proliferation rate was $97.5-96.9 \%$ in the control groups. The $p$-value was 0.476 . Therefore, the stimulation data for $3 \mathrm{mT}$ was not statistically significant.

Table 1. Statistical analyses of the cell number, proliferation rates, and $p$-values as a function of the magnetic field intensities of 3 , 5,6 and $7 \mathrm{mT}$ at $2 \mathrm{kHz}$ for 72 hours. The cell numbers were obtained from 6 wells in the same plates. The $p$-values were obtained using SPSS software.

\begin{tabular}{|c|c|c|c|c|c|c|c|}
\hline & \multicolumn{3}{|c|}{ Number of cells } & & & \multicolumn{2}{|c|}{$P$-value } \\
\hline & $\begin{array}{c}\text { Non-stimulated } \\
\text { cells }\end{array}$ & & ulated cells & \multicolumn{2}{|c|}{ Proliferation rates $(\%)$} & $\begin{array}{c}\text { Kruskal-wallis } \\
\text { test }\end{array}$ & $\begin{array}{c}\text { Mann-whitney U } \\
\text { test }\end{array}$ \\
\hline \multirow{4}{*}{$3 \mathrm{mT}$} & \multirow{2}{*}{$167,593 \pm 12 \%$} & Right & $157,222 \pm 16 \%$ & 93.81 & \multirow{2}{*}{97.57} & \multirow{2}{*}{0.701} & \multirow{4}{*}{0.476} \\
\hline & & Left & $169,815 \pm 16 \%$ & 101.33 & & & \\
\hline & \multirow{2}{*}{$188,889 \pm 5 \%$} & Right & $170,741 \pm 3 \%$ & 90.39 & \multirow{2}{*}{96.96} & \multirow{2}{*}{0.003} & \\
\hline & & Left & $195,556 \pm 4 \%$ & 103.53 & & & \\
\hline \multirow{6}{*}{$5 \mathrm{mT}$} & \multirow{2}{*}{$188,519 \pm 12 \%$} & Right & $122,222 \pm 13 \%$ & 64.83 & \multirow{2}{*}{64.73} & \multirow{2}{*}{0.005} & \multirow{6}{*}{0.000} \\
\hline & & Left & $121,852 \pm 21 \%$ & 64.64 & & & \\
\hline & \multirow{2}{*}{$167,407 \pm 10 \%$} & Right & $80,370 \pm 25 \%$ & 48.01 & \multirow{2}{*}{50.44} & \multirow{2}{*}{0.003} & \\
\hline & & Left & $88,519 \pm 13 \%$ & 52.88 & & & \\
\hline & \multirow{2}{*}{$174,444 \pm 10 \%$} & Right & $117,593 \pm 16 \%$ & 67.41 & \multirow{2}{*}{64.86} & \multirow{2}{*}{0.003} & \\
\hline & & Left & $108,704 \pm 22 \%$ & 62.31 & & & \\
\hline \multirow{2}{*}{$6 \mathrm{mT}$} & \multirow{2}{*}{$174,074 \pm 10 \%$} & Right & $132,963 \pm 20 \%$ & 76.38 & \multirow{2}{*}{80.43} & \multirow{2}{*}{0.008} & \multirow{2}{*}{0.003} \\
\hline & & Left & $147,037 \pm 4 \%$ & 84.47 & & & \\
\hline \multirow{4}{*}{$7 \mathrm{mT}$} & \multirow{2}{*}{$183,333 \pm 14 \%$} & Right & $172,222 \pm 17 \%$ & 93.94 & \multirow{2}{*}{90.05} & \multirow{2}{*}{0.385} & \multirow{4}{*}{0.049} \\
\hline & & Left & $157,963 \pm 18 \%$ & 86.16 & & & \\
\hline & \multirow{2}{*}{$184,444 \pm 10 \%$} & Right & $151,481 \pm 17 \%$ & 82.13 & \multirow{2}{*}{87.20} & \multirow{2}{*}{0.082} & \\
\hline & & Left & $170,185 \pm 16 \%$ & 92.27 & & & \\
\hline
\end{tabular}




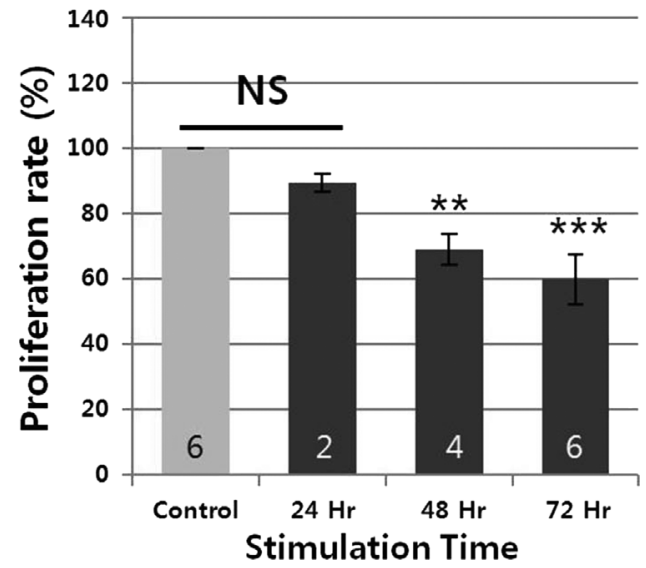

Fig. 5. The proliferation rates as a function of stimulation time for 24, 48 and 72 hours at the field intensity of $5 \mathrm{mT}$ and $2 \mathrm{kHz}$. The $p$-values were $* * p<0.01$, and $* * * p<0.001$ and the number noted below each bar is the number of repetitions.

However, it is worth noting the cell numbers in the three repeat experiments at $5 \mathrm{mT}$. The cell numbers of the control group was almost the same as $1.67-1.88 \times 10^{5}$ at 3 $\mathrm{mT}$, but the stimulated cell groups decreased dramatically to $1.22-0.8 \times 10^{5}$. The average proliferation rates significantly decreased to $64.8-50.49 \%$ and the p-values were $0.003-0.005$ for the Kruskal-wallis test. For the Mann-Whitney U test, the $p$-value was 0.000 . This result distinctly showed that the proliferation of human cancer cells was significantly suppressed by stimulation with an alternative magnetic field at $5 \mathrm{mT}$ for 72 hours.

The proliferation rates of the cell groups exposed to magnetic stimulation at 6 and $7 \mathrm{mT}$ increased to 80.4$90 \%$. The $p$-valves were 0.003 and 0.49 , respectively. We expected the proliferation rate to decrease with the increase in the magnetic field intensity, but we obtained opposite results. The biological mechanism underlying the increase in the cell proliferation at $7 \mathrm{mT}$ cannot be fully explained. However, we accept that the effect of magnetic field stimulation on the cells has an optimum condition to maintain homeostasis in living cells. Although the magnetic stimulation effect is unclear, it can be concluded that alternative magnetic stimulation of human breast cancer cells at $5 \mathrm{mT}$ and $2 \mathrm{kHz}$ distinctly affects cell proliferation.

Figure 5 shows the time dependence of stimulation on BT-20 breast cancer cells stimulated with a magnetic field of $2 \mathrm{kHz}$ and $5 \mathrm{mT}$ for 72 hours. The 24 hour cells were exposed to magnetic stimulation for 24 hours and then cultured for 48 hours without stimulation, and the 48 hour cells received 48 hours of stimulation and 24 hours of culturing without stimulation. The 72 hour cells were stimulated the entire time. All cell counting was done at the same time after 72 hours. The 24 hour stimulated cells were suppressed by up to $89 \%$, while the 48 hour cells were suppressed up to $73 \%$, and the 72 hour cells up to $60 \%$ for. Therefore, the proliferation rate decreased as the stimulation time increased.

\section{Conculsion}

The proliferation effect of human breast cancer cells as a function of alternative magnetic field intensity and stimulation time at $2 \mathrm{kHz}$ was investigated. The proliferation rate decreased dramatically up to $40 \%$ at $5 \mathrm{mT}$ under 72-hour stimulation. On the other hand, the cells were not influenced by magnetic field stimulation at 7 $\mathrm{mT}$. It is not easy to explain our results based on the physiology of cell membranes, which have various ionic flows at ion channels. However, it is important to study the effect of magnetic field intensity and frequency on cell stimulus to develop a therapeutic modality for biomedical applications.

\section{Acknowledgement}

This research was supported by Sangji university and the Basic Science Research Program through the National Research Foundation of Korea (NRF) funded by the Ministry of Education (NRF-2013R1A1A2010352).

\section{References}

[1] D. Fixler, S. Yitzhaki, A. Axelrod, T. Zinman, and A. Shainberg, Bioelectromagnetics 33, 634 (2012).

[2] D. Dallari, M. Fini, G. Giavaresi, N. Del Piccolo, C. Stagni, L. Amendola, N. Rani, S. Gnudi, and R. Giardino, Bioelectromagnetics 30, 423 (2009).

[3] T. Wang, Y. Nie, S. Zhao, Y. Han, Y. Du, and Y. Hou, Bioelectromagnetics 32, 443 (2011).

[4] I. Hilger, W. Andrä, R. Hergt, R. Hiergeist, H. Schubert, and W. A. Kaiser, Radiology 218, 570 (2001).

[5] X. Wang, Y. Chen, C. Huang, X. Wang, L. Zhao, X. Zhang, and J. Tang, Bioelectromagnetics 34, 95 (2013).

[6] J. Liang, A. W. Mok, Y. Zhu, and J. Shi, Bioelectrochemistry 94, 61 (2013).

[7] M. A. Vander Molen, H. J. Donahue, C. T. Rubin, and K. J. McLeod, Bone 27, 227 (2000).

[8] M. T. Tsai, W. J. Li, R. S. Tuan, and W. H. Chang, J. Orthopaedic Research 27, 1169 (2009).

[9] T. S. Jang, J. Y. Lee, H. S. Lee, S. Kim, and D. G. Hwang, J. Magnetics 17, 42 (2012).

[10] C. N. Gutt, Z. G. Kim, D. Hollander, and T. Bruttel, Clinical Cancer Research 9, 1474 (2003).

[11] N. J. Salkind, Encyclopedia of Measurement and Statistics 3, SAGE Publications, CA (2007) pp. 889-891. 